\title{
Gross, Histopathological, and Ultrastructural Features in Patients with COVID-19: A Literature Review
}

\author{
Authors: \\ *Mahreen Hussain, ${ }^{1}$ Tania Platero-Portillo, ${ }^{2}$ Olanrewaju Oni, ${ }^{3}$ Mai \\ Elzieny, ${ }^{4}$ Kaveri Malik Khera, ${ }^{5}$ Hemlata Padharia, ${ }^{6}$ Nehemias Guevara- \\ Rodriguez ${ }^{7,2}$ \\ 1. United Medical and Dental College, Karachi, Pakistan \\ 2. School of Medicine, University of El Salvador, San Salvador, El Salvador \\ 3. Department of Biological Sciences, Northern Illinois University, DeKalb, Illinois, USA \\ 4. Faculty of Medicine, Ain Shams University, Cairo, Egypt \\ 5. Santosh Medical College, Department of Clinical Microbiology, Ghaziabad, India \\ 6. Veer Surendra Sai Institute of Medical Sciences and Research, Burla, India, \\ 7. Rosales National Hospital, Internal Medicine Department, San Salvador, El Salvador \\ *Correspondence to mahreenhussain7@gmail.com
}

Disclosure: $\quad$ The authors have declared no conflicts of interest.

Received: $\quad 03.08 .20$

Accepted: $\quad 01.12 .20$

Keywords: $\quad$ Autopsy, biopsy, coronavirus disease (COVID-19), gross, histopathology.

Citation: $\quad$ EMJ Microbiol Infect Dis. 2021; DOI/10.33590/emjmicrobiolinfectdis/20-00195.

\begin{abstract}
The coronavirus disease (COVID-19) outbreak has led to swift efforts to learn about its clinical course, prognostic markers, and complications. Consequently, there is a lot of scattered information available regarding severe acute respiratory syndrome coronavirus-2 (SARS-CoV-2) but its pathophysiology is still poorly understood. Gross and microscopic findings are very important for understanding any disease, including COVID-19. This literature review examines and summarises the biopsy, gross autopsy, and other histopathological findings that have been reported in various organs in COVID-19 patients to increase the understanding of the disease. Many histopathological findings in various organs were nonspecific, especially in the liver and brain, while others were particular to SARS-CoV-2. Therefore, further histopathological studies and autopsies are necessary to obtain consistent and reliable findings in those with COVID-19 to fully understand the pathogenesis of the disease and the impact it has on individual organs.
\end{abstract}

\section{INTRODUCTION}

The analysis of biological samples has always played a vital part in understanding pathological processes of diseases. As the outbreak of the coronavirus disease (COVID-19) turned into a global pandemic, new information has emerged regarding various aspects of the

disease. However, the current understanding about the precise nature and reaction patterns in various organs and tissues in response to this infection is lacking and poorly understood. In the large number of original studies available on the subject matter, it can be difficult to get a full picture of the effect that COVID-19 has on the body as a whole, as many studies show 
conflicting results. In this literature review, the authors examined and summarised the macroand micropathological findings present in each organ system in a COVID-19 patient in already published literature.

\section{METHODS}

Until 11 $1^{\text {th }}$ July 2020, PubMed and Embase were searched for related published articles. In both electronic databases, the following search strategy was applied and these keywords (in the title or abstract) were used: "COVID 19" OR "coronavirus" AND "biopsy" OR "autopsy" OR "histopathology".

\section{RESULTS}

\section{Lungs ( $n=215$ cases)}

A reported gross examination was available for 89 cases that tested positive for COVID-19. The macroscopic examination in all cases showed findings that ranged which patchy to diffuse areas of consolidation to severe and extensive suppurative infiltrates. A common finding was an increased lung weight in 70

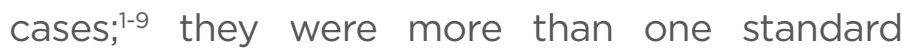
deviation heavier than average weight. Lungs consisting of firm parenchyma with regions of dark-coloured to reddish haemorrhagic areas, with focal demarcation and severe congestion were found in 38 cases. $^{3-5,7-12}$ Visible pulmonary thromboembolism were noted in 27 cases,,313-16 some of them with associated infarcts after prophylactic dose anticoagulant therapy, suggesting that pulmonary thrombi were formed despite anticoagulant therapy. ${ }^{13}$ In some cases, visibly enlarged peritracheal and peribroncheal lymph nodes were found.,10,11

Histopathological examination was available for 190 COVID-19 positive cases. The most commonly reported histopathological finding was diffuse alveolar damage, reported in all but one case ${ }^{17}$ ranging from hyaline membrane formation, alveolar wall oedema, fibrin deposits within the alveoli, severe capillary congestion and severe syncytial cell and Type II pneumocyte hyperplasia, and desquamation to diffuse necrosis of alveolar lining cells. ${ }^{2,4-6,8-13,15,18-27}$ One recently performed prospective cohort study reported that diffuse alveolar damage was the primary abnormality in hospitalised patients and almost every patient positive for severe acute respiratory syndrome coronavirus 2 (SARS-CoV-2) died without medical intervention.' Predominantly, inflammatory $\mathrm{CD} 3+$ and $\mathrm{CD} 4+\mathrm{T}$ cells were found near precapillary and postcapillary vessel walls along with macrophages and abundant polymorphonuclear leukocytes whereas the number of CD8+ T cells was lower in comparison in most cases. ${ }^{2,5,6,10,16,17,19,28,29}$ CD61+ megakaryocytes were abundantly found actively producing platelets and located within pulmonary capillaries, further indicating the stimulation of the coagulation cascade. 6,5,20

Another broad finding was pulmonary thromboembolism with fibrinous thrombi in small and large pulmonary arterioles, in some cases with haemorrhagic foci. $5,6,20-23,30$ In a comparative study, the lungs presented structurally deformed capillaries, showing abrupt calibre changes such as intussusceptive pillars within the capillaries and hallmarks of endothelial cell swelling, loss of contact with basal membrane, and disruption of intercellular junctions on electron microscopy. ${ }^{2}$ In multiple cases, ultrastructural examination showed distended cytoplasmic vacuoles within the pneumocytes with viral cytopathic changes and intracellular SARS-CoV-2. Virions were seen extracellularly among the cilia and within the cytoplasm of respiratory epithelial cells in the upper airway. 2,6,7,10,14,18,22,27 Immunohistochemistry of viral antigens was positive in the upper airway, bronchiolar and submucosal gland epithelium, Type I and II pneumocytes, alveolar macrophages, and lung hyaline membranes. ${ }^{22}$ Superimposed bronchopneumonia was described in 19 cases with mostly neutrophilic infiltrate. 4,19,20

\section{Cardiovascular system ( $n=67$ cases)}

Cardiovascular gross findings were reported in 19 autopsy cases that tested positive for COVID-19. The most commonly reported gross findings in seven cases were large vessel occlusions characterised by intimal and medial thickening with luminal narrowing. ${ }^{31-33}$ Other reported gross findings in two cases included thin myocardial trabecula, streaking of right atrial wall myocardial tissue, $^{7}$ and mottling parenchyma with a soft and rubbery texture. ${ }^{6}$ The most significant gross findings generally seen were cardiomegaly and right ventricular dilatation, which were attributed to pre-existing underlying diseases 
such as hypertension, diabetes, and obesity. No significant gross findings were observed in the remaining 10 cases.

Histopathological autopsies findings were available for 59 cases of COVID-19 positive patients. Unlike in other similar viral infections that caused viral myocarditis, there were no reported cases of COVID-19 myocarditis. The most commonly reported histopathological finding was myocyte necrosis, observed in almost all the cases except for one case. ${ }^{7}$ Changes to the myocardium included irregularity in shape with a darkened cytoplasm, though these changes were not sufficient for interpretation as acute myocardial injury in four cases; ${ }^{24}$ mild myxoid oedema; mild myocyte hypertrophy; focal nuclear pyknosis, in one case; ${ }^{7}$ and moderately enlarged cardiomyocyte with hyperchromatic nuclei and vacuolar degenerative change in three cases. ${ }^{31}$ The presence of diffuse lymphocytic endothelitis and apoptotic bodies were shown in 34 cases. ${ }^{32-35}$

A study proposed that SARS-CoV-2 might not directly impair the heart, because they observed scarce interstitial mononuclear inflammatory infiltrates in the myocardial tissue without substantial cardiac muscle tissue damage in their patient with COVID-19.36 There was also evidence of direct viral infection of endothelial cells, endothelitis with diffuse endothelial inflammation, and micro- and macrovascular thrombosis, both in the venous and arterial circulations, in 26 cases. $^{34}$

In conclusion, cardiomegaly was most likely a result of pre-existing heart disease. Although histopathological cardiac findings were notable for absence of lymphocytic myocarditis, myocyte necrosis was the most commonly reported finding.

\section{Gastrointestinal $(n=24)$}

The most common gross finding was multiple and diffuse punctate haemorrhages in the stomach $(n=3)$ and duodenal mucosa $(n=1) .^{37,38}$ Other gross findings $(n=2)$ included increased liver volume and an enlarged gallbladder. . $^{37,38}$

In all of the 24 cases, there was mild-tomoderate hepatic lobular or portal lymphocytic infiltration. 18,19,24,38,39 Four cases showed centrilobular sinusoidal dilatation. ${ }^{24}$ Minimal-tomild micro- and macrovesicular steatosis was seen in ten cases. $^{18,19,38}$ Patchy and multifocal hepatic necrosis were seen in 13 cases..$^{19,24,38}$ These findings were mostly nonspecific and can be attributed to direct viral-induced cellular injuries and potential hepatotoxicity from therapeutic drugs, or be representative of pre-existing chronic liver disease that was exacerbated during COVID-19, and COVID-19-related hyperinflammatory reactions. ${ }^{40}$ SARS-CoV-2 particles without membrane-bound vesicles in hepatocyte cytoplasm were also identified. ${ }^{38}$

\section{Central nervous system ( $n=79$ cases)}

Clearly reported gross examination was available for 41 cases that tested positive for COVID-19. The macroscopic examination showed findings that ranged from mild swelling and cerebral oedema in three cases, $, 4,41,42$ to disseminated haemorrhagic lesions throughout the cerebral white matter (size ranging from $1 \mathrm{~mm}$ to $1 \mathrm{~cm}$ ) and scattered punctate subarachnoid hemorrhages in two cases. ${ }^{41,43}$ Hydrocephalus internus was present in two cases. ${ }^{4}$ No abnormalities were seen in 23 cases apart from those which were already associated with the patients' prior comorbidities such as atherosclerosis and Parkinson's disease $(n=16){ }^{4}$

Histopathological examination was available for 65 COVID-19 positive cases. Here, 33 cases showed no microscopic abnormalities; one case mimicked glioma on imaging but on histopathological examination was negative for malignancy and positive for encephalitis. ${ }^{44}$ Reactive gliosis, neuronal satellitosis, perivascular haemorrhage, ${ }^{45}$ and disseminated haemorrhagic cerebral white matter lesions ${ }^{41}$ were found in eight, five, one, and one case, respectively. Other neuropathological findings included characteristics resembling vascular and demyelinating lesions. For example, the scattered necrotic neurons of acute hypoxic injury with red degeneration and oedema in the cerebrum (cortex), cerebellum (Purkinje cell layer), and hippocampus (CA1) were observed in 19 cases. ${ }^{41,46}$ Infarcts in brainstem, deep grey nuclei, or spinal cord were absent. In one case, the subcortical white matter had macrophage clusters with axonal injury and a perivascular acute disseminated encephalomyelitis-like appearance. ${ }^{41}$ No thrombi, vasculitis, or abnormalities of the olfactory bulb were observed in any cases. ${ }^{39,41}$ Focal leptomeningeal inflammation was detected in 
one brain specimen ${ }^{46}$ while no inflammation was present in the leptomeninges of another case. ${ }^{41}$ Perivascular lymphocytic clusters were detected in three brain specimens. ${ }^{41,46}$

\section{Kidney $(n=43)$}

The histopathological effects of COVID-19 in kidney tissues were reviewed for 43 cases. Here, 32 cases reported severe acute tubular necrosis, showing diffuse proximal tubular injury with the loss of brush border, vacuolar degeneration, mild fibrosis in the interstitium, and lymphocyte infiltration on microscopy while severe glomerular injury was absent.47-49 The vacuoles seen on light microscopy correlated with doublemembrane vesicles containing virion particles by electron microscopy. ${ }^{49}$ Infrequent haemosiderin granules, pigmented casts, and lumenobstructing red blood cell aggregates were also observed. Vasculitis, interstitial inflammation, or haemorrhage were absent. ${ }^{48}$ Electron microscopy demonstrated virus-like particles are in the tubular epithelium and podocytes. ${ }^{48}$ The virus was not only directly cytotoxic, but also had the ability to initiate CD68+ macrophage and complement C5b-9 deposition to mediate tubular pathogenesis. ${ }^{47,50}$

In a separate study that evaluated 10 patients with proteinuria and COVID-19, all renal biopsies showed renal tubular acidosis without evidence of the virus in the biopsied tissue. ${ }^{51}$

\section{Skin $(n=111)$}

The clinical presentation of skin in COVID-19 patients showed findings including urticarial, vesicular, and petechial or purpuric eruptions; erythema multiforme; vascular complications such as acro-ischaemia, livedo, necrosis, or gangrene and chilblain; and maculopapular eruptions including morbilliform rash, plaques, and pityriasis rosea. ${ }^{52}$

Histopathological examination was available for 30 COVID-19 positive cases. The most common finding, present in 18 cases, was significant degree of perivascular infiltration of the superficial dermis by lymphocytes, eosinophils, and neutrophils in vasculitic pattern with accompanying papillary oedema and extravasation of red blood cells. ${ }^{44,53-58}$ Thrombogenic vasculopathy, found in seven cases, was associated with widespread epidermal or papillary dermal or dermohypodermal and adnexal necrosis.
Adnexal necrosis spared ducts, but was present in the secretory portion of sweat coil. ${ }^{54,56,58}$ Other epidermal findings included spongiosis, basal cell vacuolation, suprabasal acantholytic ballooning keratinocytes, parakeratosis, and dyskeratotic.56,57 Superficial and deep dermal lichenoid infiltrate was also found in one case of chilblains. ${ }^{53}$ Immunohistochemistry showed extensive C5b-9 deposition within the vessels of the dermis. ${ }^{54}$

\section{Testes $(n=13)$}

Histopathological examination was available for 13 COVID-19 positive cases. Out of these, 11 cases showed swollen, vacuolated Sertoli cells. Intratubular cell detachment from basement membranes of the seminiferous tubules was noted. No inflammatory cells were found within the seminiferous tubules while the interstitium showed oedema and infiltration of $T$ lymphocytes and histiocytes. Out of the same 11 cases, $18.2 \%, 45.5 \%$, and $36.4 \%$ of cases showed $<10 \%, 10-50 \%$, and $>50 \%$ injury to seminiferous tubules, respectively, while mild to no tubular injury was present in five cases of COVID-19 negative controls. The Leydig cell count was also significantly lower than in the control group. Normal spermatogenesis was observed in all cases as ACE-2 was not expressed in spermatogonia but widely present in Sertoli and Leydig cells. ${ }^{59}$ The rest of the two cases showed orchitis. ${ }^{45}$

Table 1, 2 and 3 show the patient demographics such as sex, age, comorbidities, and country the study was conducted in.

\section{LIMITATIONS AND RECOMMENDATIONS}

The data or cases available for review were very limited as many hospitals discontinued autopsies on COVID-19 cases to limit the spread of the virus. Further, larger studies are needed to assess the effect of COVID-19 in different organs, especially on the central nervous system, kidneys, and the gastrointestinal tract. The authors also recommend that more studies are conducted in areas with higher prevalence of comorbidities, such as viral hepatitis or tuberculosis, so that the effects of specific pre-existing conditions on the outcome of the virus can be determined. Studies comparing the effects of COVID-19 on various organs in healthy patients versus in those with pre-existing conditions will also be helpful. 
Table 1: Pulmonary findings in patients with coronavirus disease (COVID-19).

\begin{tabular}{|c|c|c|c|c|c|c|}
\hline Organ & Study & $\begin{array}{l}\text { Number of } \\
\text { cases }\end{array}$ & $\begin{array}{l}\text { Country of } \\
\text { origin }\end{array}$ & Age (years) & Sex & Comorbidities \\
\hline \multirow[t]{17}{*}{ Lung } & Xu et al., ${ }^{18} 2020$ & 1 & China & 50 & Male & Lung injury, ARDS \\
\hline & $\begin{array}{l}\text { Grimes et al., }{ }^{14} \\
2020\end{array}$ & 2 & USA & Middle-age & Male & $\begin{array}{l}\text { HTN, asthma, HIV compliant } \\
\text { with antiretroviral therapy }\end{array}$ \\
\hline & $\begin{array}{l}\text { Ackerman et al., } \\
2020\end{array}$ & 7 & USA & $\begin{array}{l}\text { Mean }( \pm S D) \text { : } \\
68( \pm 9.2), \text { and } \\
80( \pm 11.5)\end{array}$ & $\begin{array}{l}\text { Female: } 2 ; \\
\text { male: } 5\end{array}$ & NA \\
\hline & $\begin{array}{l}\text { Aguiar et al., } 5 \\
2020\end{array}$ & 1 & Switzerland & 31 & Female & Morbid obesity \\
\hline & $\begin{array}{l}\text { Duarte-Neto et } \\
\text { al., }{ }^{45} 2020\end{array}$ & 10 & Brazil & NA & NA & NA \\
\hline & $\begin{array}{l}\text { Beigmohammadi } \\
\text { et al., }{ }^{19} 2020\end{array}$ & 7 & Iran & $\begin{array}{l}\text { Mean (range): } \\
67.85(46-84)\end{array}$ & $\begin{array}{l}\text { Female: } 2 ; \\
\text { male: } 5\end{array}$ & $\begin{array}{l}\text { HTN (57.0\%), RA (14.2\%), } \\
\text { none (28.5\%) }\end{array}$ \\
\hline & $\begin{array}{l}\text { Brown et al., }{ }^{17} \\
2020\end{array}$ & 1 & USA & NA & NA & NA \\
\hline & $\begin{array}{l}\text { Deshpande, et } \\
\text { al., }{ }^{13} 2020\end{array}$ & 11 & USA & NA & NA & $\begin{array}{l}\text { HTN, DM, obesity, COPD, } \\
\text { CAD, cancer, cerebrovascular } \\
\text { disease ( } 4 \text { patients), pulmonary } \\
\text { embolism ( } 1 \text { patient) }\end{array}$ \\
\hline & $\begin{array}{l}\text { Dolhnikoff et } \\
\text { al., }^{20} 2020\end{array}$ & 10 & Brazil & $\begin{array}{l}\text { Mean (range): } \\
67.8(33-83)\end{array}$ & $\begin{array}{l}\text { Female: } 5 \text {; } \\
\text { male: } 5\end{array}$ & $\begin{array}{l}\text { HTN, DM, IHD, COPD (7 } \\
\text { patients) }\end{array}$ \\
\hline & Fox et al., ${ }^{6} 2020$ & 10 & USA & Range: 44-78 & NA & $\begin{array}{l}\text { HTN, DM, obesity, } \\
\text { immunosuppressed (1 patient) }\end{array}$ \\
\hline & Jain et al.,, 2020 & NA & NA & NA & NA & NA \\
\hline & $\begin{array}{l}\text { Konopka et al., } \\
2020\end{array}$ & 8 & USA & $\begin{array}{l}\text { Average } \\
\text { (range): } 53 \\
(22-80) ; 49 \\
(33-63)\end{array}$ & $\begin{array}{l}\text { Female: } 6 \text {; } \\
\text { male: } 2\end{array}$ & $\begin{array}{l}\text { Idiopathic bronchiectasis } \\
\text { and pneumonia, suffered } \\
\text { acute intraparenchymal brain } \\
\text { haemorrhage ( } 1 \text { patient) }\end{array}$ \\
\hline & Lax et al., ${ }^{3} 2020$ & 11 & Austria & $\begin{array}{l}\text { Mean/median } \\
\text { (range): } \\
81.5 / 80.5 \\
(75-91) \\
\end{array}$ & $\begin{array}{l}\text { Female: } 3 ; \\
\text { male: } 8\end{array}$ & $\begin{array}{l}\text { HTN (81\%), DM ( } 45 \%), \\
\text { cerebrovascular disease (36\%), } \\
\text { dementia (36\%) }\end{array}$ \\
\hline & Li et al., ${ }^{40} 2020$ & NA & China & NA & NA & NA \\
\hline & $\begin{array}{l}\text { Martines et al., } 22 \\
2020\end{array}$ & 8 & USA & $\begin{array}{l}\text { Median: } 73.5 \\
\text { (two <65) }\end{array}$ & NA & $\begin{array}{l}\text { HTN (75\%), CKD (75\%), } \\
\text { cardiovascular disease (75\%), } \\
\text { obesity ( } 62.5 \%) \text {, DM (50\%) }\end{array}$ \\
\hline & $\begin{array}{l}\text { Menter et al., }{ }^{4} \\
2020\end{array}$ & 21 & Switzerland & $\begin{array}{l}\text { Mean (range): } \\
76(53-96)\end{array}$ & $\begin{array}{l}\text { Female: } 4 ; \\
\text { male: } 17\end{array}$ & $\begin{array}{l}\text { HTN (100\%), cardiovascular } \\
\text { disease ( } 71 \%) \text {, smoker (38\%), } \\
\text { pre-obesity/obesity, DM ( } 33 \%) \text {, } \\
\text { chronic neurological condition } \\
\text { ( } 23.8 \%) \text {, COPD (14.2\%), } \\
\text { malignancy (14.2\%), CLD } \\
\text { (9.5\%), CKD (19\%), acquired } \\
\text { immunosuppression (4.7\%) }\end{array}$ \\
\hline & $\begin{array}{l}\text { Pernazza et al., }{ }^{23} \\
2020\end{array}$ & 1 & Italy & 61 & Male & Lung adenocarcinoma \\
\hline
\end{tabular}


Table 1 continued.

\begin{tabular}{|c|c|c|c|c|c|c|}
\hline Organ & Study & $\begin{array}{l}\text { Number of } \\
\text { cases }\end{array}$ & $\begin{array}{l}\text { Country of } \\
\text { origin }\end{array}$ & Age (years) & Sex & Comorbidities \\
\hline & $\begin{array}{l}\text { Popa et al., } \\
2020\end{array}$ & 1 & Romania & 88 & Male & $\begin{array}{l}\text { HTN, DM, with permanent } \\
\text { electrical cardio-stimulation, } \\
\text { history of TIA, immobilised in } \\
\text { bed }\end{array}$ \\
\hline & $\begin{array}{l}\text { Scendoni et al., } \\
2020\end{array}$ & 2 & Italy & $\begin{array}{l}\text { Female: } 62 ; \\
\text { male: } 44\end{array}$ & $\begin{array}{l}\text { Female: 1; } \\
\text { male: } 1\end{array}$ & $\begin{array}{l}\text { Male: polymyositis } \\
\text { Female: HTN, autoimmune } \\
\text { hypothyroidism, DM }\end{array}$ \\
\hline & $\begin{array}{l}\text { Schaefer et al., }{ }^{16} \\
2020\end{array}$ & 7 & USA & Average: 62.4 & $\begin{array}{l}\text { Female: } 2 ; \\
\text { male: } 5\end{array}$ & $\begin{array}{l}\text { SLE, RA, pulmonary fibrosis, } \\
\text { CKD, interstitial lung disease, } \\
\text { MGUS, CAD, HTN, DM, } \\
\text { cardiomegaly, atherosclerosis, } \\
\text { dementia, status post-leg } \\
\text { amputation, COPD, NASH }\end{array}$ \\
\hline & $\begin{array}{l}\text { Shao et al., }{ }^{29} \\
2020\end{array}$ & 1 & China & 65 & Male & None \\
\hline & $\begin{array}{l}\text { Suess et al.,"1 } \\
2020\end{array}$ & 1 & Switzerland & 59 & Male & HTN, DM \\
\hline & Tian et al., ${ }^{12} 2020$ & 2 & China & $\begin{array}{l}\text { Female: } 84 ; \\
\text { male: } 73\end{array}$ & $\begin{array}{l}\text { Female: 1; } \\
\text { male: } 1\end{array}$ & $\begin{array}{l}\text { Male: HTN, DM, } \\
\text { adenocarcinoma }\end{array}$ \\
\hline & $\begin{array}{l}\text { Tian et al., }{ }^{24} \\
2020\end{array}$ & 2 & China & NA & NA & NA \\
\hline & $\begin{array}{l}\text { von der Thüsen, } \\
\text { van der Eerden }{ }^{25} \\
2020\end{array}$ & 41 & $\begin{array}{l}\text { The } \\
\text { Netherlands }\end{array}$ & NA & NA & NA \\
\hline & $\begin{array}{l}\text { Wang et al., }{ }^{26} \\
2020\end{array}$ & 2 & China & $\begin{array}{l}\text { Female: } 53 ; \\
\text { male: } 62\end{array}$ & $\begin{array}{l}\text { Female: 1; } \\
\text { male: } 1\end{array}$ & Female: DM and HTN \\
\hline & Yan et al.,7 2020 & 1 & USA & 44 & Male & Obesity \\
\hline & $\begin{array}{l}\text { Zeng et al., }{ }^{27} \\
2020\end{array}$ & 1 & China & 55 & Female & None \\
\hline & $\begin{array}{l}\text { Zhang et al., }{ }^{28} \\
2020\end{array}$ & 1 & China & 72 & Male & DM and HTN \\
\hline & $\begin{array}{l}\text { Conde et al., } \\
2020\end{array}$ & 1 & Spain & 69 & Male & None \\
\hline & $\begin{array}{l}\text { The COVID-19 } \\
\text { Autopsy, }{ }^{9} 2020\end{array}$ & 1 & Spain & 54 & Male & HTN, gout, migraine, OSA \\
\hline & $\begin{array}{l}\text { Schaller et al., }{ }^{39} \\
2020\end{array}$ & 12 & Germany & Median: 79 & $\begin{array}{l}\text { Female: } 3 ; \\
\text { male: } 7\end{array}$ & $\begin{array}{l}\text { HTN (41\%), DM (16\%), COPD } \\
\text { (16\%), malignancy (16\%), } \\
\text { obesity (16\%) }\end{array}$ \\
\hline & $\begin{array}{l}\text { Tian S et al., }{ }^{24} \\
2020\end{array}$ & 4 & China & Median: 73 & $\begin{array}{l}\text { Female: 1; } \\
\text { male: } 3\end{array}$ & DM and HTN \\
\hline
\end{tabular}

ARDS: acute respiratory distress syndrome; CAD: coronary artery disease; CKD: chronic kidney disease; CLD: chronic liver disease; COPD: chronic obstructive pulmonary disease; DM: diabetes mellitus; HTN: hypertension; IHD: ischaemic heart disease; MGUS: monoclonal gammopathy of undetermined significance; NA: not available; NASH: non-alcoholic steatohepatitis; OSA: obstructive sleep apnoea; RA: rheumatoid arthritis; SLE: systemic lupus erythematosus; TIA: transient ischaemic attack. 
Table 2: Cardiovascular findings in patients with coronavirus disease (COVID-19).

\begin{tabular}{|c|c|c|c|c|c|c|}
\hline Organ & Study & $\begin{array}{l}\text { Number of } \\
\text { cases }\end{array}$ & $\begin{array}{l}\text { Country of } \\
\text { origin }\end{array}$ & Age (years) & Sex & Comorbidities \\
\hline \multirow[t]{8}{*}{ CVS } & $\begin{array}{l}\text { Becker et al., } \\
2020\end{array}$ & 4 & USA & Mean: 50 & Male & NA \\
\hline & $\begin{array}{l}\text { Buja et al.,31 } \\
2020\end{array}$ & 4 & USA & Range: 44-76 & Female: 1; male: 3 & $\begin{array}{l}\text { Obesity, DM, } \\
\text { CKD }\end{array}$ \\
\hline & $\begin{array}{l}\text { Centurión et } \\
\text { al., } 362020\end{array}$ & 15 & Paraguay & NA & NA & NA \\
\hline & $\begin{array}{l}\text { Craver et al., }{ }^{35} \\
2020\end{array}$ & 1 & USA & 17 & Male & NA \\
\hline & Fox et al.., ${ }^{6} 2020$ & 10 & USA & Range: 44-78 & NA & $\begin{array}{l}\text { HTN, obesity, } \\
\text { DM }\end{array}$ \\
\hline & $\begin{array}{l}\text { Pons et al., } 34 \\
2020\end{array}$ & 26 & France & Mean: 65 & NA & NA \\
\hline & $\begin{array}{l}\text { Tian et al.,24 } \\
2020\end{array}$ & 4 & China & Range: 59-81 & Female: 1; male: 3 & Pneumonia \\
\hline & $\begin{array}{l}\text { Varga et al.,33 } \\
2020\end{array}$ & 3 & Switzerland & Range: 58-71 & Female: 1; male: 2 & $\begin{array}{l}\text { HTN, renal } \\
\text { transplant } \\
\text { patient }\end{array}$ \\
\hline
\end{tabular}

CKD: chronic kidney disease; CVS: cardiovascular system; DM: diabetes mellitus; HTN: hypertension; NA: not available.

Table 3: Findings in other organ systems in patients with coronavirus disease (COVID-19) (gastrointestinal tract, central nervous system, renal, skin, testes).

\begin{tabular}{|c|c|c|c|c|c|c|}
\hline Organ & Study & $\begin{array}{l}\text { Number of } \\
\text { cases }\end{array}$ & $\begin{array}{l}\text { Country of } \\
\text { origin }\end{array}$ & Age (years) & Sex & Comorbidities \\
\hline \multirow[t]{6}{*}{ GIT } & $\begin{array}{l}\text { Wang et al., } \\
2020\end{array}$ & 156 & China & Mean: 51 & Male: 82 & $\begin{array}{l}\text { DM, HTN, digestive system } \\
\text { disease, endocrine system } \\
\text { disease, nervous system } \\
\text { disease, chronic respiratory } \\
\text { disease }\end{array}$ \\
\hline & $\begin{array}{l}\text { Schaller et al., } \\
2020\end{array}$ & 10 & Germany & $\begin{array}{l}\text { Mean (range): } \\
79(64-90)\end{array}$ & Male: 7 & $\begin{array}{l}\text { CAD, COPD, CKD, DM, morbid } \\
\text { obesity, endocrine system } \\
\text { disease, CML, adenocarcinoma } \\
\text { of the lung }\end{array}$ \\
\hline & $\begin{array}{l}\text { Beigmohammadi } \\
\text { et al., }{ }^{19} 2020\end{array}$ & 7 & Iran & $\begin{array}{l}\text { Mean (range): } \\
67.9(46-84)\end{array}$ & Male: 5 & $\begin{array}{l}\text { DM, HTN, CAD, digestive } \\
\text { system disease, RA }\end{array}$ \\
\hline & Xu et al.. ${ }^{27} 2020$ & 1 & China & 50 & Male: 1 & NA \\
\hline & $\begin{array}{l}\text { Adachi et al., } 37 \\
2020\end{array}$ & 1 & Japan & 84 & Female: 1 & NA \\
\hline & $\begin{array}{l}\text { Tian et al., }{ }^{24} \\
2020\end{array}$ & 4 & China & $\begin{array}{l}\text { Mean (range): } \\
73(59-81)\end{array}$ & Male: 3 & $\begin{array}{l}\text { CLL, CLD, HTN, DM, renal } \\
\text { transplantation }\end{array}$ \\
\hline CNS & $\begin{array}{l}\text { Duarte-Neto et } \\
\text { al., }^{20} 2020\end{array}$ & 9 & Brazil & $\begin{array}{l}\text { Median } \\
\text { (range): } \\
69(33-83)\end{array}$ & NA & NA \\
\hline
\end{tabular}


Table 3 continued.

\begin{tabular}{|c|c|c|c|c|c|c|}
\hline Organ & Study & $\begin{array}{l}\text { Number of } \\
\text { cases }\end{array}$ & $\begin{array}{l}\text { Country of } \\
\text { origin }\end{array}$ & Age (years) & Sex & Comorbidities \\
\hline & $\begin{array}{l}\text { Menter et al., }{ }^{4} \\
2020\end{array}$ & 18 & Switzerland & $\begin{array}{l}\text { Median } \\
\text { (range): } 76 \\
(53-96)\end{array}$ & $\begin{array}{l}\text { Female: } 4 ; \\
\text { male: } 17\end{array}$ & $\begin{array}{l}\text { HTN, CAD, DM, ESRD, obesity, } \\
\text { chronic neurological condition, } \\
\text { CLD }\end{array}$ \\
\hline & $\begin{array}{l}\text { Reichard et al., } \\
2020\end{array}$ & 1 & USA & 71 & Male: 1 & CAD \\
\hline & Liu et al., ${ }^{42} 2020$ & 1 & NA & NA & NA & NA \\
\hline & $\begin{array}{l}\text { Bradley et al., } 43 \\
2020\end{array}$ & 14 & USA & $\begin{array}{l}\text { Median } \\
\text { (range): } 74 \\
(42-84)\end{array}$ & $\begin{array}{l}\text { Female: } 8 ; \\
\text { male: } 6\end{array}$ & HTN, CKD, OSA, DM, obesity \\
\hline & Efe et al..,4 2020 & 1 & NA & 35 & Female: 1 & None \\
\hline & $\begin{array}{l}\text { Solomon et al.., } \\
2020\end{array}$ & 18 & NA & $\begin{array}{l}\text { Median } \\
\text { (interquartile } \\
\text { range): } 62 \\
(53-75)\end{array}$ & $\begin{array}{l}\text { Female: } 4 ; \\
\text { male: } 14\end{array}$ & $\begin{array}{l}\text { DM (12 patients), HTN (11), CAD } \\
\text { (5), hyperlipidaemia (5), CKD } \\
\text { (4), prior stroke (4), dementia } \\
\text { (4), treated anaplastic } \\
\text { astrocytoma (1) }\end{array}$ \\
\hline & $\begin{array}{l}\text { Schaller et al., }{ }^{39} \\
2020\end{array}$ & 10 & Germany & $\begin{array}{l}\text { Median } \\
\text { (range): } 79 \\
(64-90)\end{array}$ & Male: 7 & $\begin{array}{l}\text { Leukaemia, HTN, } \\
\text { hypothyroidism, COPD, CKD, } \\
\text { cardiomyopathy, obesity, DM, } \\
\text { atrial fibrillation, CAD }\end{array}$ \\
\hline \multirow[t]{4}{*}{ Kidney } & Su et al., ${ }^{48} 2020$ & 26 & China & 69 & $\begin{array}{l}\text { Female: 7; } \\
\text { male: } 19\end{array}$ & $\begin{array}{l}\text { Renal failure, multiple organ } \\
\text { dysfunction, clinical signs of } \\
\text { kidney injury (nine patients) }\end{array}$ \\
\hline & $\begin{array}{l}\text { Diao B et al., }{ }^{47} \\
2020\end{array}$ & 6 & China & Range: 21-92 & NA & HTN (three patients) \\
\hline & $\begin{array}{l}\text { Farkash EA et } \\
\text { al., }{ }^{49} 2020\end{array}$ & 1 & China & 53 & Male: 1 & $\begin{array}{l}\text { Aortic dissection repair } \\
\text { complicated by renal failure, } \\
\text { obesity, hyperlipidaemia }\end{array}$ \\
\hline & $\begin{array}{l}\text { Sharma P et al., } \\
2020\end{array}$ & 10 & China & Mean: 66 & $\begin{array}{l}\text { Female: 5; } \\
\text { male: } 5\end{array}$ & DM, HTN \\
\hline \multirow[t]{7}{*}{ Skin } & $\begin{array}{l}\text { Duarte-Neto et } \\
\text { al., }^{20} 2020\end{array}$ & 12 & Brazil & $\begin{array}{l}\text { Median } \\
\text { (range): } \\
69(33-83)\end{array}$ & NA & NA \\
\hline & $\begin{array}{l}\text { Kolivras et al., } 53 \\
2020\end{array}$ & 1 & NA & 23 & Male: 1 & Psoriasis \\
\hline & $\begin{array}{l}\text { Magro et al., }{ }^{54} \\
2020\end{array}$ & 5 & NA & Range: 32-73 & $\begin{array}{l}\text { Female: } 2 ; \\
\text { male: } 3\end{array}$ & $\begin{array}{l}\text { Females: no comorbidities } \\
\text { Males: CAD, DM, heart failure, } \\
\text { ESRD, obesity }\end{array}$ \\
\hline & $\begin{array}{l}\text { Diaz-Guimaraens } \\
\text { et al.. }{ }^{55} 2020\end{array}$ & 1 & Spain & 48 & Male: 1 & HTN \\
\hline & $\begin{array}{l}\text { Gianotti et al., } \\
2020\end{array}$ & 3 & Italy & Range: 57-89 & $\begin{array}{l}\text { Female: } 2 \text {; } \\
\text { male: } 1\end{array}$ & NA \\
\hline & $\begin{array}{l}\text { Ahouach et al.,57 } \\
2020\end{array}$ & 1 & NA & 57 & Female: 1 & NA \\
\hline & $\begin{array}{l}\text { Llamas-Velasco } \\
\text { et al., }{ }^{58} 2020\end{array}$ & 1 & NA & 61 & Male: 1 & DM \\
\hline
\end{tabular}


Table 3 continued.

\begin{tabular}{|l|l|l|l|l|l|l|}
\hline Organ & Study & $\begin{array}{l}\text { Number of } \\
\text { cases }\end{array}$ & $\begin{array}{l}\text { Country of } \\
\text { origin }\end{array}$ & Age (years) & Sex & Comorbidities \\
\hline Testes & $\begin{array}{l}\text { Duarte-Neto et } \\
\text { al., } 2020\end{array}$ & 2 & Brazil & $\begin{array}{l}\text { Median } \\
\text { (range): } \\
69(33-83)\end{array}$ & Male: 1 & NA \\
\cline { 2 - 6 } & $\begin{array}{l}\text { Yang et al.,59 } \\
2020\end{array}$ & 11 & $\begin{array}{l}\text { Mean (range): } \\
65(42-87)\end{array}$ & Male: 1 & $\begin{array}{l}\text { HTN, CKD, CAD, neoplasia (two } \\
\text { patients had no comorbidities) }\end{array}$ \\
\hline
\end{tabular}

CAD: coronary artery disease; CKD: chronic kidney disease; CLD: chronic liver disease; CLL: chronic lymphocytic leukaemia; CML: chronic myelogenous leukaemia; CNS: central nervous system; COPD: chronic obstructive pulmonary disease; DM: diabetes mellitus; ESRD: end-stage renal disease; GIT: gastrointestinal tract; HTN: hypertension; NA: not available; OSA: obstructive sleep apnea; RA: rheumatoid arthritis.

\section{CONCLUSION}

In conclusion, the many findings in multiple SARS-CoV-2 as many patients had underlying pre-existing conditions; therefore, further caseorgans could not be definitively attributed to histopathological findings to SARS-CoV-2.

\section{References}

1. Konopka $\mathrm{K}$ et al. Diffuse alveolar damage (DAD) from coronavirus disease 2019 infection is morphologically indistinguishable from other causes of DAD. Histopathology. 2020;doi:10.1111/ his.14180. [Epub ahead of print]

2. Ackerman $\mathrm{M}$ et al. Pulmonary vascular endothelialitis, thrombosis, and angiogenesis in COVID-19. N Engl J Med. 2020;383(2):120-8.

3. Lax AF et al. Pulmonary arterial thrombosis in COVID-19 with fatal outcome: results from a prospective, single-center, clinicopathologic case series. Ann Intern Med. 2020;173(5):350-61.

4. Menter T et al. Post-mortem examination of COVID-19 patients reveals diffuse alveolar damage with severe capillary congestion and variegated findings of lungs and other organs suggesting vascular dysfunction. Histopathology. 2020;77(2):198-209.

5. Aguiar $D$ et al. Inside the lungs of COVID-19 disease. Int J Legal Med. 2020; doi:10.1007/s00414-020-023189. [Epub ahead of print].

6. Fox E et al. Pulmonary and cardiac pathology in African American patients with COVID-19: an autopsy series from New Orleans. Lancet Respir Med. 2020;8(7):681-6.
7. Yan L et al. COVID-19 in a Hispanic woman. Arch Pathol Lab Med. 2020;144(9):1041-7.

8. Navarro Conde et al. Autopsy findings from the first known death from severe acute respiratory syndrome SARS-CoV-2 in Spain. Rev Esp Patol. 2020;53(7):188-92

9. The Autopsy Project. The first COVID-19 autopsy in Spain performed during the early stages of the pandemic. Rev Esp Patol. 2020;53(3):182-7.

10. Popa MF et al. Virus-associated hemophagocytic lymphohistiocytosis - the severe course expression in SARS-COV-2 infection? Rom J Leg Med. 2020;1(28):1-7.

11. Suess C, Hausmann R. Gross and histopathological pulmonary findings in a COVID-19 associated death during self-isolation. Int J Legal Med. 2020;doi:10.1007/s00414-020-023198. [Epub ahead of print].

12. Tian $\mathrm{S}$ et al. Pulmonary pathology of early-phase 2019 novel coronavirus (COVID-19) pneumonia in two patients with lung cancer. J Thorac Oncol. 2020;15(5):700-4.

13. Deshpande C. Thromboembolic findings in COVID-19 autopsies: pulmonary thrombosis or embolism? Ann Intern Med. 2020;doi:10.7326/ M20-3255.
14. Grimes Z et al. Fatal pulmonary thromboembolism in SARS CoV-2-infection. Cardiovasc Pathol. 2020;doi:10.1016/j. carpath.2020.107227.

15. Scendoni R et al. Histopathology of COVID-19 pneumonia in two nononcological, non-hospitalised cases as a reliable diagnostic benchmark. Diagn Pathol. 2020;15(1):73.

16. Schaefer IM et al. In situ detection of SARS-CoV-2 in lungs and airways of patients with COVID-19. Mod Pathol. 2020;33:2104-14.

17. Brown R et al. Morphoproteomics and etiopathogenic features of pulmonary COVID-19 with therapeutic implications: a case study. Ann Clin Lab Sci. 2020;50(3):308-13.

18. $\mathrm{Xu} \mathrm{Z}$ et al. Pathological findings of COVID-19 associated with acute respiratory distress syndrome. Lancet Respir Med. 2020;8(4):420-2.

19. Beigmohammadi MT et al. Pathological findings of postmortem biopsies from lung, heart, and liver of 7 deceased COVID-19 patients. Int J Surg Pathol. 2020;doi:10.1177/1066896 920935195. [Epub ahead of print].

20. Dolhnikoff $M$ et al. Pathological evidence of pulmonary thrombotic phenomena in severe COVID-19. J Throm Haemos. 2020;18(6):1517-9. 
21. Jain A. COVID-19 and lung pathology. Indian J Pathol Microbiol. 2020;63(2):171-2.

22. Martines $\mathrm{R}$ et al. Pathology and pathogenesis of SARS-CoV-2 associated with fatal coronavirus disease, United States. Emerg Infect Dis. 2020;26(9):2005-15.

23. Pernazza A et al. Early histologic findings of pulmonary SARSCoV-2 infection detected in a surgical specimen. Virchows Arch. 2020; doi:10.1007/s00428-020-028291. [Epub ahead of print].

24. Tian $\mathrm{S}$ et al. Pathological study of the 2019 novel coronavirus disease (COVID-19) through postmortem core biopsies. Mod Pathol. 2020;33(6):1007-14

25. von der Thüsen JH, van der Eerden M. Histopathology and genetic susceptibility in COVID-19 pneumonia. Eur J Clin Invest. 2020;50(7):e13259.

26. Wang $\mathrm{C}$ et al. Alveolar macrophage dysfunction and cytokine storm in the pathogenesis of two severe COVID-19 patients. EBioMedicine. 2020;57:102833.

27. Zeng $Z$ et al. Pulmonary pathology of early phase COVID-19 pneumonia in a patient with a benign lung lesion. Histopathology. 2020;77(5):823-31.

28. Zhang $\mathrm{H}$ et al. Histopathologic changes and SARS-COV-2 immunostaining in the lung of a patient with COVID-19. Ann Intern Med. 2020;172(9):629-32.

29. Shao $\mathrm{C}$ et al. Evolution of severe acute respiratory syndrome coronavirus 2 RNA test results in a patient with fatal coronavirus disease 2019: a case report. Hum Pathol. 2020;1010:82-8.

30. Monteiro RAA et al. Histologicalultrasonographical correlation of pulmonary involvement in severe COVID-19. Intensive Care Med 2020;46(9):1766-8.

31. Buja LM et al. The emerging spectrum of cardiopulmonary pathology of the coronavirus disease: report of 3 autopsies from Houston, Texas, and review of autopsy findings from other United States Cities. Cardiovasc Pathol. 2020;48:107233.

32. Becker R. COVID-19 update: COVID19-associated coagulopathy. J Thromb Thrombolysis. 2020;50(1):5467.

33. Varga $Z$ et al. Endothelial cell infection and endotheliitis in COVID-19. Lancet. 2020;395(10234):1417-8.
34. Pons S et al. The vascular endothelium: the cornerstone of organ dysfunction in severe SARS-COV-2 infection. Crit Care. 2020;24(1):353.

35. Craver R et al. Fatal eosinophilic myocarditis in a healthy 17-year-old male with severe acute respiratory syndrome coronavirus 2 (SARSCoV-2c). Fetal Pediatr Pathol. 2020;39(3):263-8

36. Centurión OA et al. Potential mechanisms of cardiac injury and common pathways of inflammation in patients with COVID-19. Crit Pathw Cardiol. 2020;doi:10.1097/ HPC.0000000000000227.

37. Adachi T et al. Clinicopathologic and immunohistochemical findings from autopsy of patient with COVID-19, Japan. Emerg Infect Dis. 2020;26(9):2157-61.

38. Wang $Y$ et al. SARS-CoV-2 infection of the liver directly contributes to hepatic impairment in patients with COVID-19. J Hepatol. 2020;73(4):80716.

39. Schaller T et al. Postmortem examination of patients with COVID-19. JAMA. 2020;323(24):251820.

40. Li Y, Xiao SY. Hepatic involvement in COVID-19 patients: pathology, pathogenesis, and clinical implications. J Med Virol. 2020;92(9):1491-4.

41. Reichard RR et al. Neuropathology of COVID-19: a spectrum of vascular and acute disseminated encephalomyelitis (ADEM)-like pathology. Acta Neuropathologica. 2020;140(1):1-6.

42. Liu $Q$ et al. Gross examination report of a COVID-19 death autopsy. Fa Yi Xue Za Zhi. 2020;36:21-3.

43. Bradley BT et al. Histopathology and ultrastructural findings of fatal COVID-19 infections. medRxiv. 2020;doi:https://doi.org/10.1101/2020. 04.17.20058545.

44. Efe IE et al. COVID-19-associated encephalitis mimicking glial tumor. World Neurosurg. 2020;140:46-8.

45. Duarte-Neto A et al. Pulmonary and systemic involvement of COVID-19 assessed by ultra-soundguided minimally invasive autopsy. Histopathology. 2020;77(2):186-97.

46. Solomon IH et al. Neuropathological features of Covid-19. N Engl J Med. 2020;383(10):989-92

47. Diao B et al. Human kidney is a target for novel severe acute respiratory syndrome coronavirus 2 (SARSCoV-2) infection. 2020;doi:https://doi. org/10.1101/2020.03.04.20031120.

48. Su $\mathrm{H}$ et al. Renal histopathological analysis of 26 postmortem findings of patients with COVID-19 in China. Kidney Int. 2020;98(1):219-27.

49. Farkash EA et al. Ultrastructural evidence for direct renal infection with SARS-COV-2. J Am Soc Nephrol. 2020;31(8):1683-87.

50. Staico MF et al. The kidney in COVID-19: protagonist or figurant? Panminerva Med. 2020;doi:10.23736/ S0031-0808.20.03965-8. [Epub ahead of print].

51. Sharma P et al. COVID-19-associated kidney injury: a case series of kidney biopsy findings. J Am Soc Nephrol. 2020;31(9):1948-58

52. Criado PR et al. Are the cutaneous manifestations during or due to SARS-CoV-2 infection/COVID-19 frequent or not? Revision of possible pathophysiologic mechanisms. Inflamm Res. 2020;69(8):745-56

53. Kolivras A et al. Coronavirus (COVID-19) infection-induced chilblains: a case report with histopathologic findings. JAAD Case Rep. 2020;6(6):489-92

54. Magro $\mathrm{C}$ et al. Complement associated microvascular injury and thrombosis in the pathogenesis of severe COVID-19 infection: a report of five cases. Transl Res. 2020;doi:10.1016/j.trsl.2020.04.007.

55. Diaz-Guimaraens B et al. Petechial skin rash associated with severe acute respiratory syndrome coronavirus 2 infection. JAMA Dermatol. 2020;156(7):820-2.

56. Gianotti R et al. Cutaneous clinico-pathological findings in three COVID-19-positive patients observed in the metropolitan area of Milan, Italy. Acta Derm Venereol. 2020;100(8):adv00124.

57. Ahouach B et al. Cutaneous lesions in a patient with COVID-19: are they related? $\mathrm{Br} J$ Dermatol. 2020;183(2):e31.

58. Llamas-Velasco $M$ et al. Thrombotic occlusive vasculopathy in skin biopsy from a livedoid lesion of a COVID-19 patient. Br J Dermatol. 2020;18:(3):591-3.

59. Yang $M$ et al. Pathological findings in the testes of COVID-19 patients: clinical implications. EU Urol Focus. 2020;6(5):1124-9. 